\title{
CMB and Cosmological Parameters: Current Status and Prospects.
}

\author{
Alessandro Melchiorri* \\ Universita' di Roma "La Sapienza" \\ Ple Aldo Moro 2, 00185, Rome, Italy
}

\begin{abstract}
The last years have been an exciting period for the field of the Cosmic Microwave Background (CMB) research. With recent CMB satellite, balloon-borne and ground-based experiments we are entering a new era of 'precision' cosmology that enables us to use the $\mathrm{CMB}$ anisotropy measurements to constrain the cosmological parameters and test new theoretical scenarios.
\end{abstract}

\section{Introduction}

The nature of cosmology as a mature and testable science lies in the realm of observations of Cosmic Microwave Background (CMB) anisotropy and polarization. The recent highquality measurements of the CMB anisotropies provided by ground-based, balloon-borne and satellite experiments have indeed presented cosmologists with the possibility of studying the large scale properties of our universe with unprecedented precision.

An increasingly complete cosmological image arises as the key parameters of the cosmological model have now been constrained within a few percent accuracy. The impact of these results in different sectors than cosmology has been extremely relevant since CMB studies can set stringent constraints on the early thermal history of the universe and its particle content. For example, important constraints have been placed in fields related to particle physics or quantum gravity like neutrino physics, extra dimensions, and super-symmetry theories.

In the next couple of years, new and current on-going experiments will provide datasets with even higher quality and information. In particular, accurate measurements of the CMB polarization statistical properties represent a new research area. The CMB polarization has been detected by two experiments, but remains to be thoroughly investigated. In conjunction with our extensive knowledge about the CMB temperature anisotropies, new constraints on the physics of the early universe (gravity waves, isocurvature perturbations,

\footnotetext{
${ }^{*}$ Speaker.
} 
variations in fundamental constants) as well as late universe phenomena (reionization, formation of the first objects, galactic foregrounds) will be investigated with implications for different fields ranging from particle physics to astronomy.

Moreover, new CMB observations at small (arcminute) angular scales will probe secondary fluctuations associated with the first nonlinear objects. This is where the first galaxies and the first quasars may leave distinct imprints in the CMB and where an interface between cosmology and the local universe can be established. In this proceedings I will briefly review the current status of CMB observations, I discuss the agreement with the current theoretical scenario and I will finally draw some conclusions.

\section{The standard picture.}

The standard model of structure formation (described in great detail in several reviews,

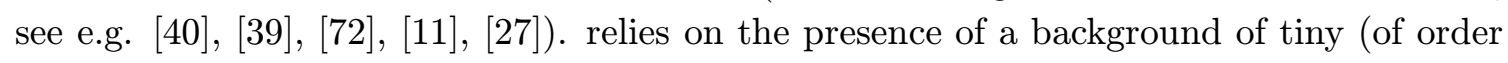
$10^{-6}$ ) primordial density perturbations on all scales (including those larger than the causal horizon).

This primordial background of perturbations is assumed gaussian, adiabatic, and nearly scale-invariant as generally predicted by the inflationary paradigm. Once inflation is over, the evolution of all Fourier mode density perturbations is linear and passive (see [2] $\left.\overline{2}_{1} \overline{7}_{1}\right)$.

Moreover, prior to recombination, a given Fourier mode begins oscillating as an acoustic wave once the horizon overtakes its wavelength. Since all modes with a given wavelength begin evolving simultaneously the resulting acoustic oscillations are phase-coherent, leading to a structure of peaks in the temperature and polarization power spectra of the Cosmic

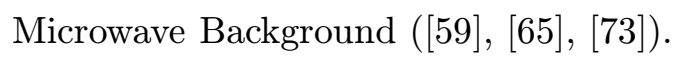

The anisotropy with respect to the mean temperature $\Delta T=T-T_{0}$ of the CMB sky in the direction $\mathbf{n}$ measured at time $t$ and from the position $\vec{x}$ can be expanded in spherical harmonics:

$$
\frac{\Delta T}{T_{0}}(\mathbf{n}, t, \vec{x})=\sum_{\ell=2}^{\infty} \sum_{m=-\ell}^{m=\ell} a_{\ell m}(t, \vec{x}) Y_{\ell m}(\mathbf{n})
$$

If the fluctuations are Gaussian all the statistical information is contained in the 2point correlation function. In the case of isotropic fluctuations, this can be written as:

$$
\left\langle\frac{\Delta T}{T_{0}}\left(\mathbf{n}_{1}\right) \frac{\Delta T}{T_{0}}\left(\mathbf{n}_{2}\right)\right\rangle=\frac{1}{4 \pi} \sum_{\ell}(2 \ell+1) C_{\ell} P_{\ell}\left(\mathbf{n}_{1} \cdot \mathbf{n}_{2}\right) .
$$

where the average is an average over "all the possible universes" i.e., by the ergodic theorem, over $\vec{x}$. The CMB power spectrum $C_{\ell}$ are the ensemble average of the coefficients $a_{\ell m}$,

$$
C_{\ell}=\left\langle\left|a_{\ell m}\right|^{2}\right\rangle
$$

A similar approach can be used for the cosmic microwave background polarization and the cross temperature-polarization correlation functions. Since it is impossible to measure $\frac{\Delta T}{T_{0}}$ in every position in the universe, we cannot do an ensemble average. This introduces a 


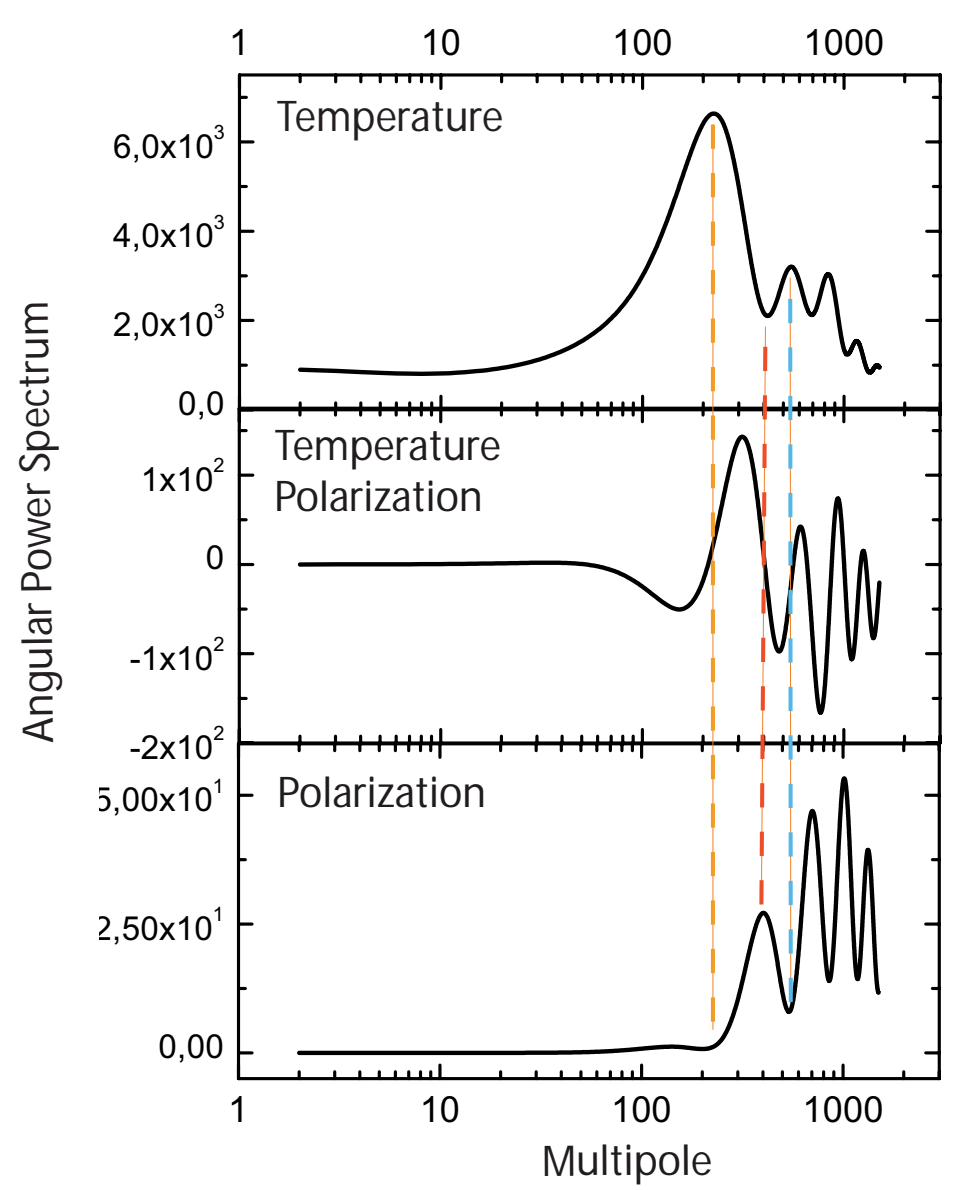

Figure 1: Theoretical predictions for the CMB temperature, polarization and cross temperaturepolarization power spectra in the case of the standard model of structure formation. The peaks in the temperature and polarization spectra are alternate.

fundamental limitation for the precision of a measurement (the cosmic variance) which is important especially for low multipoles. If the temperature fluctuations are Gaussian, the $C_{\ell}$ have a chi-square distribution with $2 \ell+1$ degrees of freedom and the observed mean deviates from the ensemble average by

$$
\frac{\Delta C_{\ell}}{C_{\ell}}=\sqrt{\frac{2}{2 \ell+1}}
$$

Moreover, in a real experiment, one never obtain complete sky coverage because of the limited amount of observational time (ground based and balloon borne experiments) or because of galaxy foreground contamination (satellite experiments). All the telescopes also have to deal with the noise of the detectors and are obviously not sensitive to scales smaller than the angular resolution.

In Figure 1 we plot the theoretical prediction for CMB temperature and polarization power 


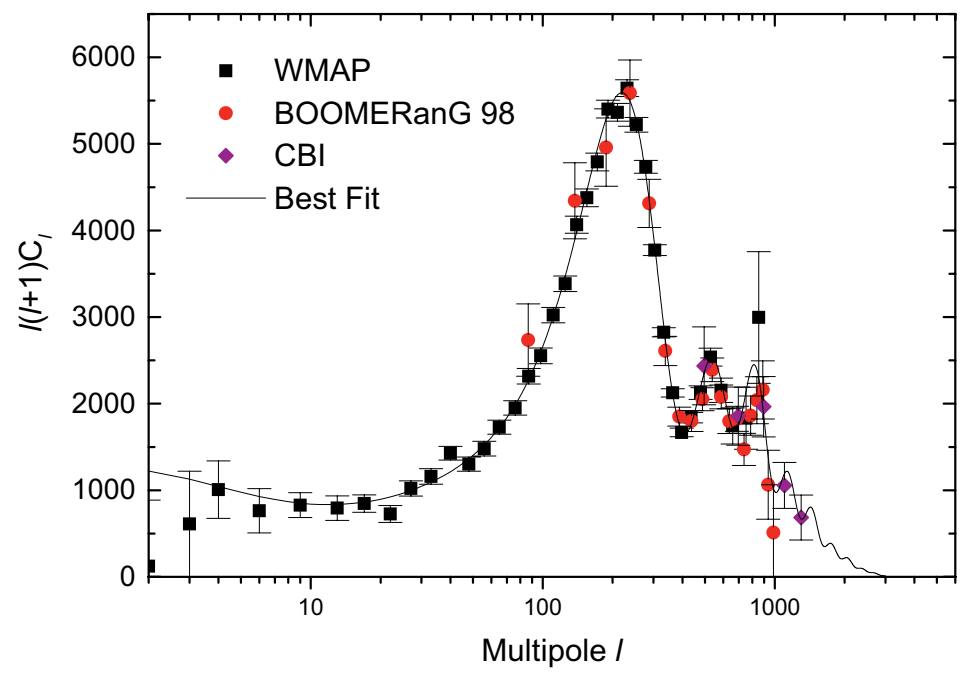

Figure 2: Cosmic Microwave Anisotropies data from WMAP, BOOMERanG and CBI experiments and the theoretical best fit. There is an excellent agreement between data and theory.

spectra and the cross-correlation between temperature and polarization in the case of the so-called 'concordance' model. The power spectra show an unique structure: The temperature power spectrum is flat on large scale while shows oscillations on smaller scales. The polarization and cross temperature-polarization spectra are also showing oscillations on smaller scales, but the signal at large scale is expected to be negligible.

Different mechanisms are responsible for the oscillations on small scale. On the scale of the thickness of the last scattering surface, temperature anisotropies are more coupled to density and gravity perturbations, while polarization is more coupled to velocity perturbations. Gravity and density perturbations obey a cosine function, while velocity perturbations follow a sine function. The most striking observational prediction of this is the out-of-phase position of the peaks and dips in the temperature and polarization power spectra. The peaks and dips of the cross-correlation spectra fall in the middle (see e.g. [3]

The shape of the power spectra depends on the value of the cosmological parameters assumed in the theoretical computation. Since the overall picture must be consistent, the cosmological parameters determined indirectly from CMB observations must agree with the values inferred from independent observations (Big Bang Nucleosynthesis, Galaxy Surveys, Ly- $\alpha$ forest clouds, simulations, etc etc.).

\section{The latest measurements.}

The last years have been an exciting period for the field of the CMB research. With the

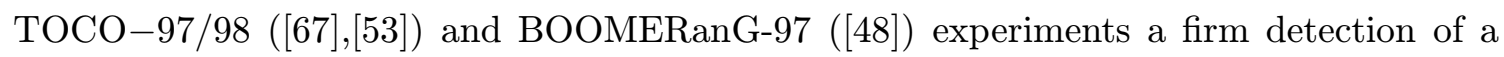
first peak in the CMB angular power spectrum on about degree scales has been obtained. In the framework of adiabatic Cold Dark Matter (CDM) models, the position, amplitude and width of this peak provide strong supporting evidence for the inflationary predictions 


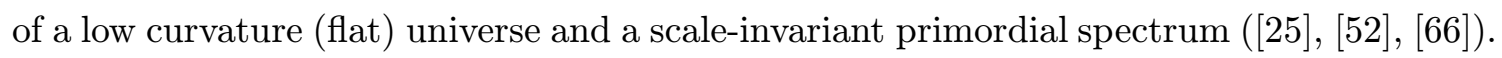

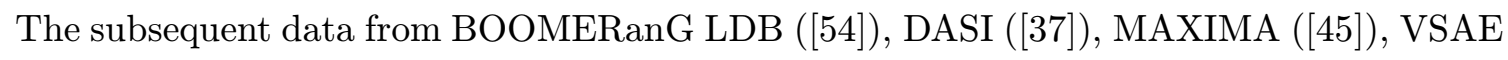

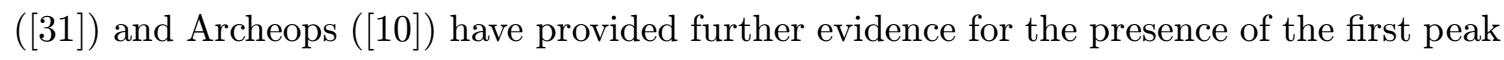
and refined the data at larger multipole hinting towards the presence of multiple peaks in

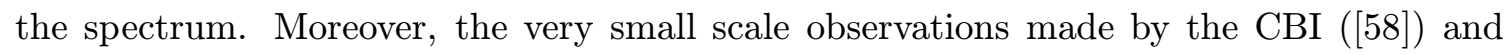

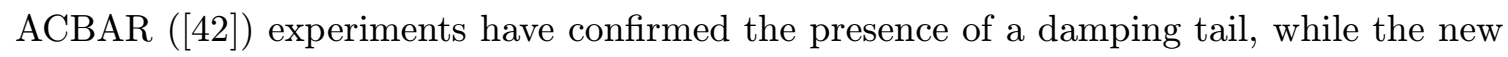
DASI results presented the first evidence for polarization ([i] $\left.\bar{i}_{-} \overline{1}_{1}\right)$.

The combined data clearly confirmed the model prediction of acoustic oscillations in the primeval plasma and shed new light on several cosmological and inflationary parameters (

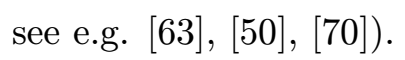

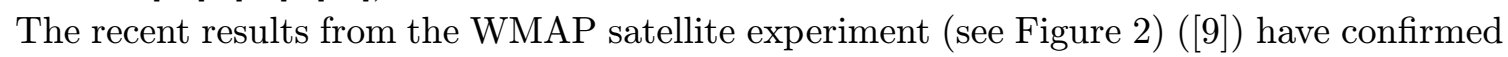
in a spectacular way all these previous results with a considerable reduction of the error bars. In particular, the amplitude and position of the first two peaks in the spectrum are

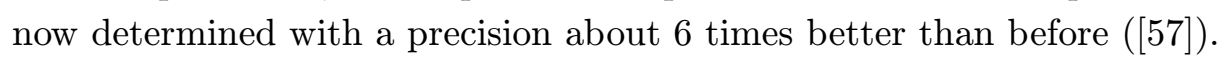

Furthermore, the WMAP team released the first high quality measurements of the temperaturepolarization spectrum [i]3i]. In particular. WMAP detected large angular scale polarization, indicative of an earlier reionization of the intergalactic medium ([3] $\left.\overline{3}_{-1}\right)$. The presence of polarization at intermediate angular scale helps in discriminating inflationary models from

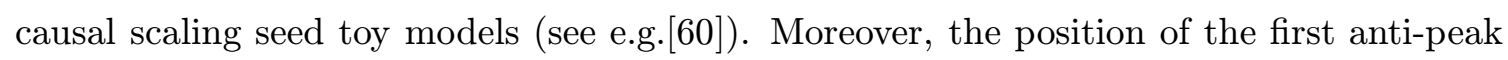
and second peak in the spectrum are also in agreement with the prediction of inflation

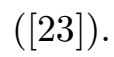

As main intriguing discrepancy, the WMAP data shows (in agreement with the previous COBE data) a lower temperature quadrupole than expected. The statistical significance

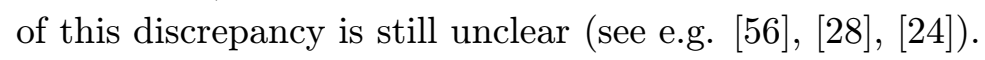

The CMB anisotropies measured by WMAP are also in good agreement with the standard inflationary prediction of gaussianity $\left(\left[{ }^{3} \overline{4} \bar{L}_{i}\right]\right)$.

\section{CMB constraints on the standard model.}

In principle, the standard scenario of structure formation based on adiabatic primordial fluctuations can depend on more than 11 parameters.

However for a first analysis and under the assumption of a flat universe which is already well consistent with CMB data, it is possible to restrict ourselves to just 5 parameters: the tilt of primordial spectrum of scalar perturbations $n_{S}$, the optical depth of the universe $\tau_{c}$, the physical energy densities in baryons and dark matter $\omega_{b}=\Omega_{b} h^{2}$ and $\omega_{d m}=\Omega_{d m} h^{2}$ and the Hubble parameter $h$.

In Table 1 we report the constraints on these parameters obtained by the WMAP team

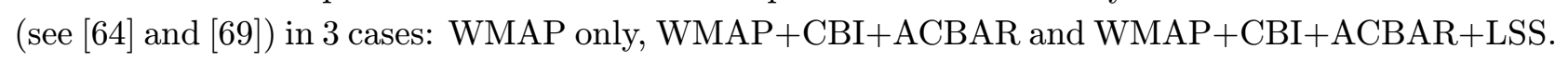
Also, for comparison, we present the CMB+LSS results previous to WMAP in the forth column.

As we can see a value for the baryon density $\omega_{b}=0.020 \pm 0.002$ as predicted by Standard

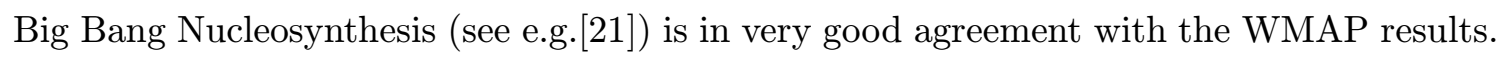
The WMAP data is in agreement with the previous results and its inclusion reduces the 


\begin{tabular}{lllll}
\hline & WMAP & WMAPext & WMAPext+LSS & Pre-WMAP+LSS \\
\hline$\Omega_{b} h^{2}$ & $0.024 \pm 0.001$ & $0.023 \pm 0.001$ & $0.023 \pm 0.001$ & $0.021 \pm 0.003$ \\
\hline$\Omega_{m} h^{2}$ & $0.14 \pm 0.02$ & $0.13 \pm 0.01$ & $0.134 \pm 0.006$ & $0.14 \pm 0.02$ \\
\hline$h$ & $0.72 \pm 0.05$ & $0.73 \pm 0.05$ & $0.73 \pm 0.03$ & $0.69 \pm 0.07$ \\
\hline$n_{s}$ & $0.99 \pm 0.04$ & $0.97 \pm 0.03$ & $0.97 \pm 0.03$ & $0.97 \pm 0.04$ \\
\hline$\tau_{c}$ & $0.166_{-0.071}^{+0.076}$ & $0.143_{-0.062}^{+0.071}$ & $0.148_{-0.071}^{+0.073}$ & $0.07_{-0.05}^{+0.07}$ \\
\hline
\end{tabular}

Table 1: Current constraints on the 5 parameters of the standard model (flat universe). The WMAP results are taken from Spergel et al. 2003, the previous results are taken from Melchiorri and Odman 2003 (see also Slosar et al. 2003 and Wang et al. 2003).

error bar on this parameter by a factor 3 .

The amount of cold dark matter is also well constrained by the CMB data. The presence of power around the third peak is crucial in this sense, since it cannot be easily accommodated in models based on just baryonic matter (see e.g. [י] we can see, including the CMB data on those scales (not sampled by WMAP) halves the error bars. WMAP is again in agreement with the previous determination and its inclusion reduces the error bar on this parameter by of factor 3-4.

These values implies the existence of a cosmological constant at high significance with $\Omega_{\Lambda}=0, \Omega_{M}=1$ excluded at 5- $\sigma$ from the WMAP data alone and at $\sim 15-\sigma$ when combined with supernovae data. A cosmological constant is also suggested from the evidence

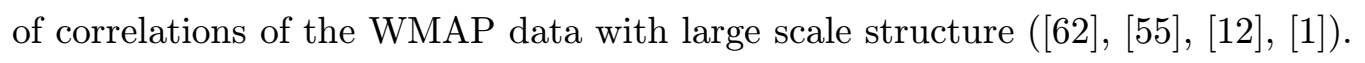

Under the assumption of flatness, is possible to constrain the value of the Hubble parameter $h$. The constraints on this parameter are in very good agreement with the HST constraint $([\overline{2} \overline{\underline{g}} \overline{\underline{1}}])$.

An increase in the optical depth $\tau_{c}$ after recombination by reionization damps the amplitude of the CMB peaks. This small scale damping can be somewhat compensated by an increase in the spectral index $n_{S}$. This leads to a nearly perfect degeneracy between $n_{S}$ and $\tau_{c}$ and, in practice, no significant upper bound on these parameters can be placed from temperature data. However, large scale polarization data, as measured by WMAP, can break this degeneracy. At the same time, inclusion of galaxy clustering data can determine $n_{S}$ and further break the degeneracy. As we can see, the current constraint on the spectral index is close to scale invariance $\left(n_{S} \sim 1\right)$ as predicted by inflation. The best-fit value of the optical depth determined by WMAP is slightly higher but consistent in between $1-\sigma$ with supercomputer simulations of reionization processes $\left(\tau_{c} \sim 0.10\right.$, see e.g. [i] $\left.\left[\begin{array}{l}{[} \\ \overline{1}\end{array}\right]\right)$.

\section{Constraints on possible extensions of the standard model.}

The standard model provides a reasonable fit to the data. However it is possible to consider several modifications characterized by the inclusion of new parameters. The data considered here doesn't show any definite evidence for those modifications, providing more a set of useful constraints. The present status can be briefly summarized as follows:

- Running of the Spectral Index 
The possibility of a scale dependence of the scalar spectral index, $n_{S}(k)$, has been

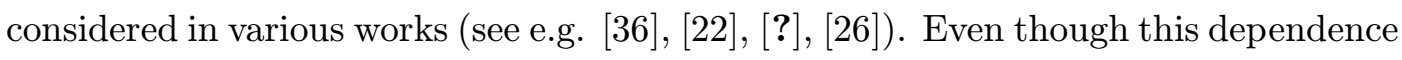
is considered to have small effects on CMB scales in most of the slow-roll inflationary models, it is worthwhile to see if any useful constraint can be obtained. The present

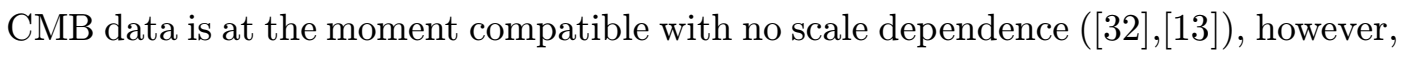
joint analyses with other datasets (like Lyman- $\alpha$ ) shows a $\sim 2-\sigma$ evidence for a negative running $([\overline{6} \overline{0} \overline{0}])$. At the moment, the biggest case against running comes from reionization models, which are unable to reach the large optical depth observed

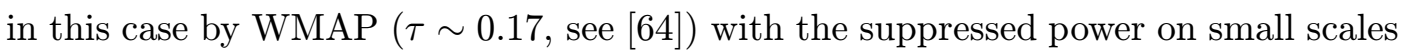

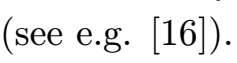

- Gravitational Waves.

The metric perturbations created during inflation belong to two types: scalar perturbations, which couple to the stress-energy of matter in the universe and form the "seeds" for structure formation and tensor perturbations, also known as gravitational wave perturbations. A sizable background of gravity waves is expected in most of the inflationary scenarios and a detection of the GW background can provide information on the second derivative of the inflaton potential and shed light on the physics at $\sim 10^{16} \mathrm{Gev}$.

The amplitude of the GW background is weakly constrained by the current CMB data. However, when information from BBN, local cluster abundance and galaxy clustering are included, an upper limit of about $r=C_{2}^{T} / C_{2}^{S}<0.5$ (no running) is

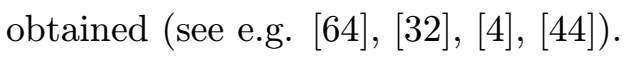

- Isocurvature Perturbations

Another key assumption of the standard model is that the primordial fluctuations were adiabatic. Adiabaticity is not a necessary consequence of inflation though and many inflationary models have been constructed where isocurvature perturbations

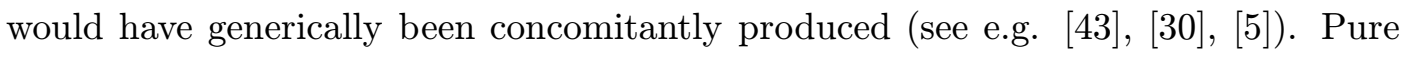
isocurvature perturbations are highly excluded by present CMB data $\left(\left[{ }^{6} 0_{0}^{i}\right]\right)$. Due to degeneracies with other cosmological parameters, the amount of isocurvature modes

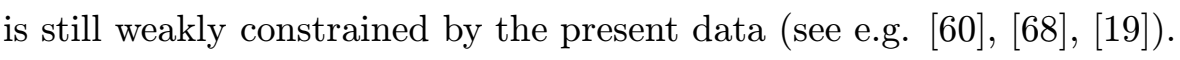

- Modified recombination.

The standard recombination process can be modified in several ways. Extra sources of ionizing and resonance radiation at recombination or having a time-varying finestructure constant, for example, can delay the recombination process and leave an imprint on the CMB anisotropies. The present data is in agreement with the standard recombinations scheme. However, non-standard recombination scenarios are still consistent with the current data and may affect the current WMAP constraints on inflationary parameters like the spectral index, $n_{s}$, and its running (see e.g. [i:ini],

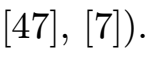


- Neutrino Physics

The effective number of neutrinos and their effective mass can be both constrained by combining cosmological data. The combination of present cosmological data under the assumption of several priors provide a constraint on the effective neutrino mass of $m_{e e}<0.23 \mathrm{eV}$ ( $\left.\left.6 \overline{6} \overline{4} \overline{4}\right]\right)$. The data constraints the effective number of neutrino species

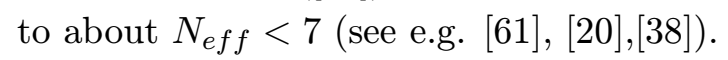

- Dark Energy and its equation of state

The discovery that the universe's evolution may be dominated by an effective cosmological constant is one of the most remarkable cosmological findings of recent years. Observationally distinguishing a time variation in the equation of state or finding it different from -1 is a powerful test for the cosmological constant. The present constraints on $w$ obtained combining the CMB data with several other cosmological datasets are consistent with $w=-1$, with models with $w<-1$ slightly preferred

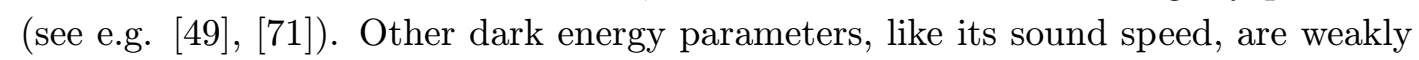

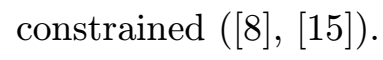

\section{Conclusions}

The recent CMB data represent a beautiful success for the standard cosmological model. The acoustic oscillations in the CMB temperature power spectrum, a major prediction of the model, have now been detected with high statistical significance. The amplitude and shape of the cross correlation temperature-polarization power spectrum is also in agreement with the expectations.

Furthermore, when constraints on cosmological parameters are derived under the assumption of adiabatic primordial perturbations their values are in agreement with the predictions of the theory and/or with independent observations.

The largest discrepancy between the standard predictions and the data seems to come from the low value of the CMB quadrupole. New physics has been proposed to explain

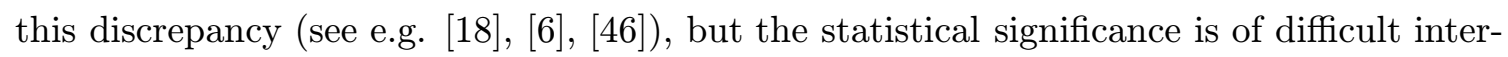
pretation.

As we saw in the previous section modifications as extra background of relativistic particles, isocurvature modes or non-standard recombination schemes are still compatible with current CMB observations, but are not necessary and can be reasonably constrained when complementary datasets are included.

\section{Acknowledgements}

I wish to thank the organizers of the conference, Jose' Furtado Valle and Sergio Pastor in particular. Many thanks also to Rachel Bean, Will Kinney, Rocky Kolb, Carlos Martins, Laura Mersini, Roya Mohayaee, Carolina Oedman, Antonio Riotto, Graca Rocha, Joe Silk, and Mark Trodden for comments, discussions and help. 


\section{References}

[1] N. Afshordi, Y. S. Loh and M. A. Strauss, arXiv:astro-ph/0308260.

[2] A. Albrecht, D. Coulson, P.G. Ferreira and J. Magueijo, Phys. Rev. Lett. 76, 1413 (1996).

[3] P. P. Avelino et al., Phys. Rev. D 64 (2001) 103505 [arXiv:astro-ph/0102144].

[4] V. Barger, H. S. Lee and D. Marfatia, Phys. Lett. B 565 (2003) 33 [arXiv:hep-ph/0302150].

[5] N. Bartolo, S. Matarrese and A. Riotto, Phys. Rev. D 64 (2001) 123504 [arXiv:astro-ph/0107502].

[6] M. Bastero-Gil, K. Freese and L. Mersini-Houghton, index," arXiv:hep-ph/0306289.

[7] R. Bean, A. Melchiorri and J. Silk, Phys. Rev. D 68 (2003) 083501 [arXiv:astro-ph/0306357].

[8] R. Bean and O. Dore, arXiv:astro-ph/0307100.

[9] C. L. Bennett et al., Astrophys. J. Suppl. 148 (2003) 1 [arXiv:astro-ph/0302207].

[10] A. Benoit et al. [Archeops Collaboration], Astron. Astrophys. 399 (2003) L19 [arXiv:astro-ph/0210305].

[11] J. R. Bond, Class. Quant. Grav. 15 (1998) 2573.

[12] S. Boughn and R. Crittenden, arXiv:astro-ph/0305001.

[13] S. L. Bridle, A. M. Lewis, J. Weller and G. Efstathiou, Mon. Not. Roy. Astron. Soc. 342 (2003) L72 [arXiv:astro-ph/0302306].

[14] S. Burles, K. M. Nollett and M. S. Turner, Astrophys. J. 552, L1 (2001) [arXiv:astro-ph/0010171].

[15] R. R. Caldwell and M. Doran, arXiv:astro-ph/0305334.

[16] R. Cen, Astrophys. J. 591 (2003) L5 [arXiv:astro-ph/0303236].

[17] B. Ciardi, A. Ferrara and S. D. M. White, Mon. Not. Roy. Astron. Soc. 344 (2003) L7 [arXiv:astro-ph/0302451].

[18] C. R. Contaldi, M. Peloso, L. Kofman and A. Linde, JCAP 0307 (2003) 002 [arXiv:astro-ph/0303636].

[19] P. Crotty, J. Garcia-Bellido, J. Lesgourgues and A. Riazuelo, Phys. Rev. Lett. 91 (2003) 171301 [arXiv:astro-ph/0306286].

[20] P. Crotty, J. Lesgourgues and S. Pastor, Phys. Rev. D 67 (2003) 123005 [arXiv:astro-ph/0302337].

[21] R. H. Cyburt, B. D. Fields and K. A. Olive, Phys. Lett. B 567 (2003) 227 [arXiv:astro-ph/0302431].

[22] E. J. Copeland, I. J. Grivell and A. R. Liddle, arXiv:astro-ph/9712028.

[23] S. Dodelson, AIP Conf. Proc. 689 (2003) 184 [arXiv:hep-ph/0309057].

[24] O. Dore, G. P. Holder and A. Loeb, arXiv:astro-ph/0309281.

[25] S. Dodelson and L. Knox, Phys. Rev. Lett. 84, 3523 (2000) [arXiv:astro-ph/9909454].

[26] S. Dodelson and E. Stewart, arXiv:astro-ph/0109354. 
[27] R. Durrer, arXiv:astro-ph/0109522.

[28] G. Efstathiou, arXiv:astro-ph/0310207.

[29] W. Freedman et al., Astrophysical Journal, 553, 2001, 47.

[30] C. Gordon, D. Wands, B. A. Bassett and R. Maartens, Phys. Rev. D 63 (2001) 023506 [arXiv:astro-ph/0009131].

[31] K. Grainge et al., Mon. Not. Roy. Astron. Soc. 341 (2003) L23 [arXiv:astro-ph/0212495].

[32] W. H. Kinney, E. W. Kolb, A. Melchiorri and A. Riotto, arXiv:hep-ph/0305130.

[33] A. Kogut et al., Astrophys. J. Suppl. 148 (2003) 161 [arXiv:astro-ph/0302213].

[34] E. Komatsu et al., Astrophys. J. Suppl. 148 (2003) 119 [arXiv:astro-ph/0302223].

[35] A. Kosowsky, arXiv:astro-ph/9811163.

[36] A. Kosowsky and M. S. Turner, Phys. Rev. D 52 (1995) 1739 [arXiv:astro-ph/9504071].

[37] N. W. Halverson et al., arXiv:astro-ph/0104489.

[38] S. Hannestad, JCAP 0305 (2003) 004 [arXiv:astro-ph/0303076].

[39] W. Hu, D. Scott, N. Sugiyama and M. J. White, Phys. Rev. D 52, 5498 (1995) [arXiv:astro-ph/9505043].

[40] W. Hu, N. Sugiyama and J. Silk, Nature 386, 37 (1997) [arXiv:astro-ph/9604166].

[41] J. Kovac, E. M. Leitch, P. C., J. E. Carlstrom, H. N. W. and W. L. Holzapfel, Nature 420 (2002) 772 [arXiv:astro-ph/0209478].

[42] C. 1. Kuo et al. [ACBAR collaboration], arXiv:astro-ph/0212289.

[43] D. Langlois and A. Riazuelo, Phys. Rev. D 62 (2000) 043504.

[44] S. M. Leach and A. R. Liddle, arXiv:astro-ph/0306305.

[45] A. T. Lee et al., Astrophys. J. 561 (2001) L1 [arXiv:astro-ph/0104459].

[46] J. P. Luminet, J. Weeks, A. Riazuelo, R. Lehoucq and J. P. Uzan, Nature 425 (2003) 593 [arXiv:astro-ph/0310253].

[47] C. J. A. Martins, A. Melchiorri, G. Rocha, R. Trotta, P. P. Avelino and P. Viana, arXiv:astro-ph/0302295.

[48] P. D. Mauskopf et al. [Boomerang Collaboration], Astrophys. J. 536, L59 (2000) [arXiv:astro-ph/9911444].

[49] A. Melchiorri, L. Mersini, C. J. Odman and M. Trodden, Phys. Rev. D 68 (2003) 043509 [arXiv:astro-ph/0211522].

[50] A. Melchiorri and C. Odman, Phys. Rev. D 67 (2003) 081302 [arXiv:astro-ph/0302361].

[51] A. Melchiorri and J. Silk, arXiv:astro-ph/0203200.

[52] A. Melchiorri et al. [Boomerang Collaboration], Astrophys. J. 536 (2000) L63 [arXiv:astro-ph/9911445].

[53] A. D. Miller et al., Astrophys. J. 524, L1 (1999) [arXiv:astro-ph/9906421].

[54] C. B. Netterfield et al. [Boomerang Collaboration], arXiv:astro-ph/0104460. 
[55] M. R. Nolta et al., arXiv:astro-ph/0305097.

[56] A. de Oliveira-Costa, M. Tegmark, M. Zaldarriaga and A. Hamilton, arXiv:astro-ph/0307282.

[57] L. Page et al., arXiv:astro-ph/0302220.

[58] T. J. Pearson et al., Astrophys. J. 591 (2003) 556 [arXiv:astro-ph/0205388].

[59] P.J.E. Peebles, and Yu, J.T. 1970, Ap.J. 162, 815

[60] H. V. Peiris et al., Astrophys. J. Suppl. 148 (2003) 213 [arXiv:astro-ph/0302225].

[61] E. Pierpaoli, Mon. Not. Roy. Astron. Soc. 342 (2003) L63 [arXiv:astro-ph/0302465].

[62] R. Scranton et al. [SDSS Collaboration], arXiv:astro-ph/0307335.

[63] A. Slosar et al., Mon. Not. Roy. Astron. Soc. 341 (2003) L29 [arXiv:astro-ph/0212497].

[64] D. N. Spergel et al., Astrophys. J. Suppl. 148 (2003) 175 [arXiv:astro-ph/0302209].

[65] Sunyaev, R.A. \& Zeldovich, Ya.B., 1970, Astrophysics and Space Science 7, 3

[66] M. Tegmark, Astrophys. J. 514, L69 (1999) [arXiv:astro-ph/9809201].

[67] E. Torbet et al., Astrophys. J. 521, L79 (1999) [arXiv:astro-ph/9905100].

[68] J. Valiviita and V. Muhonen, Phys. Rev. Lett. 91 (2003) 131302 [arXiv:astro-ph/0304175].

[69] L. Verde et al., Astrophys. J. Suppl. 148 (2003) 195 [arXiv:astro-ph/0302218].

[70] X. Wang, M. Tegmark, B. Jain and M. Zaldarriaga, arXiv:astro-ph/0212417.

[71] J. Weller and A. M. Lewis, arXiv:astro-ph/0307104.

[72] M. J. White, D. Scott and J. Silk, Ann. Rev. Astron. Astrophys. 32 (1994) 319.

[73] M. L. Wilson and J. Silk, Astrophys. J. 243 (1981) 14. 